\title{
A Qualitative Insight into Barriers to Tuberculosis Case Detection in East Gojjam Zone, Ethiopia
}

\author{
Mulusew Andualem Asemahagn, ${ }^{1 \star}$ Getu Degu Alene, ${ }^{1}$ and Solomon Abebe Yimer $^{2,3}$ \\ ${ }^{1}$ School of Public Health, College of Medicine and Health Sciences, Bahir Dar University, Bahir Dar, Ethiopia; ${ }^{2}$ Department of Microbiology, Unit for \\ Genome Dynamics, Faculty of Medicine, University of Oslo, Oslo, Norway; ${ }^{3}$ Coalition for Epidemic Preparedness Innovations (CEPI), Oslo, Norway
}

\begin{abstract}
Tuberculosis (TB) remains to be the leading cause of morbidity and mortality in the developing world. Early TB case detection (TCD) and treatment of infectious cases is vital to reduce the TB burden. The objective of this study was to identify possible barriers to TCD in East Gojjam Zone, northwest Ethiopia. The study used a descriptive phenomenological research method. The study participants included 21 TB patients, six TB control officers, and 40 health workers (HWs) selected by a heterogeneous purposive sampling technique. In-depth interviews and focus group discussions were used to collect data. Interviews were audio recorded, transcribed verbatim, translated, and thematically analyzed using NVivo 12 software (developed by QSR International Qualitative Software Developer, Melbourne, Australia). The study participants identified numerous barriers to TCD which were grouped into three major themes and 14 subthemes: 1) patient-related barriers including rural residence, low income, poor health literacy, and health-seeking delay; 2) healthcare system barriers grouped into two subthemes: HWs barriers (shortage of HWs, lack of training access, and low level of knowledge and skills) and health facility barriers (health service delay, using only passive TCD strategy, poor health education provision, and lack of regular supervision and timely feedback); 3) sociocultural and environmental barriers which included stigma and discrimination, lack of health information sources, poor transportation infrastructure, and community resistance. In conclusion, the TCD activity which is one of the pillars of the TB control program has been confronted with several patient-related, environmental, and healthcare system-related barriers. Improving community health literacy, scale-up access, and improving quality of TB diagnostic services, conducting regular supportive supervision and provision of timely feedback, arranging regular refresher training and staff motivation and recruitment schemes, and engaging local health officials and political leaders to address budgetary problems for TB and transportation infrastructure challenges are imperative interventions to enhance the TCD efforts in the study area.
\end{abstract}

\section{INTRODUCTION}

Tuberculosis (TB) is among the leading causes of morbidity and mortality in the developing world. The WHO reported 10 million new cases and 1.5 million deaths globally in $2018 .{ }^{1}$ The 30 high-TB burden countries collectively contributed to $87 \%$ of the global TB burden where $24 \%$ of the cases were from African countries. ${ }^{1}$ To reduce the high TB burden, countries have been implementing the WHO-recommended TB control strategies, at various time points; directly observed treatment short-course (DOTS); and Stop TB and End TB strategies in 1993/1994, 2006, and 2015, respectively. However, although there has been a general trend of decline in the global TB incidence, the reduction has been so slow, particularly in the African region because of various factors including poverty, ${ }^{1-4}$ high TB/HIV/DM comorbidities, drug-resistant TB (MDRTB), ${ }^{1,5}$ poor access to quality healthcare services, ${ }^{4,6}$ and low level of community awareness about TB., 3-10

Ethiopia has adopted the WHO-recommended TB control strategy since 1994. The country has also been implementing the health extension program (primary healthcare services at the community level) and involved the private health sector Public-Private Mix DOTS to enhance the TB control activities and achieve the national case detection and treatment. ${ }^{11}$ Despite all the TB control efforts, however, TB continues to pose a major public health threat in Ethiopia. Currently, the country is one of the 30 high-TB, TB/HIV and-MDR TB burden countries with a TB incidence rate of $151 / 100,000$ population. The magnitude of TB-HIV coinfection and MDR-TB was reported to be $7 / 100,000$ and $1.4 / 100,000$ populations, respectively. ${ }^{1}$

* Address correspondence to Mulusew Andualem Asemahagn, School of Public Health, College of Medicine and Health Sciences, Bahir Dar University, P.O. Box 79, Bahir Dar, Ethiopia. E-mail: muler.hi@gmail.com
The highest priority in TB control is early identification and treatment of the untreated infectious TB cases. ${ }^{1,12}$ Delayed TB case detection (TCD) and treatment results in complications of illness and high TB transmission, which is a big challenge for the global TB control program. For instance, more than three million estimated TB patients remain undiagnosed globally. ${ }^{1}$ Similarly, both the national and regional TB case notification rates in Ethiopia are below $60 \%$, which are far from the WHO target of detecting $70 \%$ of the infectious TB cases. ${ }^{1,13}$

The TB burden and case notification rates in Ethiopia vary across regions and woredas in the country. 9,12 For example, in the Amhara region, a TB incidence rate of $177 / 100,000$ population has been recorded with a low TB case notification rate of $57 \% .{ }^{14}$ Among the 10 zones of the Amhara region, the lowest TB case notification rate (49\%) was reported from East Gojjam zone, where the current study was conducted. ${ }^{9,15}$ To date, few qualitative studies have addressed barriers to TCD from the patients' perspectives. ${ }^{16-18}$ Little is known about barriers to TCD from the perspectives of all potential stakeholders. ${ }^{12,19}$ Therefore, this study was conducted to explore the barriers to TCD related to patients, health workers (HWs), sociocultural and environment factors, and the overall healthcare system related-barriers in East Gojjam zone, Amhara region, Ethiopia.

\section{MATERIALS AND METHODS}

Study design. The study adopted a descriptive phenomenological research approach to explore the views and experiences of TB patients, TB control program officers, and HWs on the barriers to TCD. This design is appropriate to understand the perceptions, perspectives, and understandings of the study participants about TCD barriers from their lived experiences, routine TB control activities, and challenges they faced. $^{20-22}$ We used an inductive approach to generate 
meanings from the data and identify patterns and relationships of themes and concepts. ${ }^{20,21}$ All the approaches from study design to data analysis were carried out based on the consolidated criteria for reporting qualitative studies. ${ }^{22}$

Study period and settings. The study was conducted between September and December 2018 in East Gojjam zone, northwest Ethiopia. The zone is one of the 10 zones in the Amhara region and has an estimated population of 2,632,632, with 1,282,676 (48.7\%) males and 1,350,541 (51.3\%) females. Of the total population, $85 \%$ are rural farmers. East Gojjam zone covers an area of $14,010 \mathrm{~km}^{2}$ and is divided into 18 administrative woredas that are further divided into 49 urban and 392 rural kebeles, the lowest administrative units. At the time of data collection, there were nine hospitals, 102 health centers (HCs), 406 health posts, and nine private clinics eligible to offer TB services. ${ }^{9,15}$

Study participants and sampling procedures. Because of resource constraints and large geographic area coverage of the study zone, we randomly selected $30 \%$ of the total 18 woredas in the zone. The selected woredas included Hulet Eju Enese, Enebse Sar Midir, Dubay Telatgen, Dejen, Machakel, and Debre Markos town administration. To get a diversity of opinions on the topic, a heterogeneous purposive sampling technique was applied to recruit study participants. The criteria considered for inclusion were patients' stage of TB treatment phase, level of engagement, and experiences of HWs in TB control (greater than 6 months of working experience). Accordingly, we selected TB patients who were in the intensive phase treatment period, health extension workers (HEWs), and HWs (TB focal persons whose professions were either nurses or health officers), and outpatient and laboratory department heads of public and private health facilities from six randomly selected woredas (the fourth level of the administrative hierarchy in Ethiopia which is next to Kebeles). In places where there were more than one TB focal person per health facility, we selected a more experienced TB control focal person. Regional, zonal, woreda TB officers (governmental and nongovernmental organizations (NGOs)) were also involved in the interview. The sample size was determined by the data saturation level.

Data collection tools and procedures. Data were collected by in-depth interviews (IDIs) and focus-group discussions (FGDs) using a separate open-ended interview guide for patients and HWs. Audio tapes and field notebooks were used to collect data. The interview guide for TB patients consisted of questions about personal and clinical profiles, TB knowledge level (TB symptoms, transmission, prevention, diagnosis, and treatment), perceptions on community awareness about TB, access to TB information sources, and barriers in accessing TB diagnostic and treatment services. The interview guide for HWs included knowledge on TCD activities and TB case notification rate, perceptions about community awareness on TB (prevention, early diagnosis, and treatment), health information sources to HWs and the community, and barriers that lower TCD performance at zonal, woreda, and facility levels. Both the IDI and FGD interview materials were pretested before the actual data collection was commenced. The objectives of the pretesting were to evaluate language competency and content validity, estimate the length of full interview delivery, maximize methodological skills, and achieve proficiency standards for qualitative data collection. It helped us redesign our data collection materials for word clarity, order of questions, identifying and removing less important questions, and avoiding redundancy of questions. The average time for saturation point was 46 minutes.

The principal investigator and facility heads arranged a quiet private office in each health facility for facilitating the interviews and FGDs. Investigators conducted 27 IDIs (21 IDIs among TB patients and six IDls among TB control program officers) and six FGDs among HWs. Each FGD consisted of five to eight participants. All the interviews were conducted in Amharic, the local language, and were audio recorded after getting written informed consent from the participants. Interview assistants recorded sociodemographic information for each respondent with their code and took additional notes. Personal code with a piece of paper was given to each study participant to keep confidentiality while giving a chance to participate in the FGDs and link their audio records to their descriptions in field notes. The average interview time was 48 minutes, ranging from 40 to 80 minutes.

Data trustworthiness. Investigators collected data from different data sources: TB patients with different occupations, education level and residence, TB control program officers working at different levels (region, zone, and woreda), HWs (TB focal persons, Outpatient Department [OPD] heads, and laboratory heads) from public and private facilities, and HEWs representing different health posts. We also used two types of data collection methods (IDIs and FGDs). The interview guide was pretested before data collection started. Moreover, recording the whole research process (thick descriptions); presenting de-identified interviews, transcripts, codes, categories, themes, and descriptions/concepts/to an external experienced researcher (audit trail); and presenting draft findings to peer academic staff were used to check the validity of the research process and findings. The sample size was relatively large, and we gave an adequate data collection period of 4 months. Both the supporting and diverging responses are included in the article.

Data processing and analysis. Data transcription and analysis began alongside data collection. The process was iterative that included rechecking and evaluating all steps throughout the whole process (Figure 1). All the audio recordings were first transcribed verbatim in Amharic/local language/and then translated into English for analysis. First, the first author (M. A. A.) transcribed and translated the audio records and field notes. Second, all the authors evaluated and approved the transcripts and translations through regular discussions and reviewing the field data records focusing on answering the study objectives. As indicated by Figure 1, we used the following steps during transcription, translation, analysis, and interpretation of findings.

1. Listen and relisten the audio recordings and read and reread field notes.

2. Conduct the transcript, read, and reread the transcripts through rechecking audio records and considering memos.

3. Translate the transcripts through rechecking from the transcripts, audio records, and memos.

4. Generate the codes, subcodes, categories, themes, and subthemes through usual reading of translates, transcripts, and tape recordings.

5. Evaluate the codes, categories, and themes and recheck the former steps to correct the disagreement parts (words, contents, and concepts).

6. Approve themes and subthemes. 


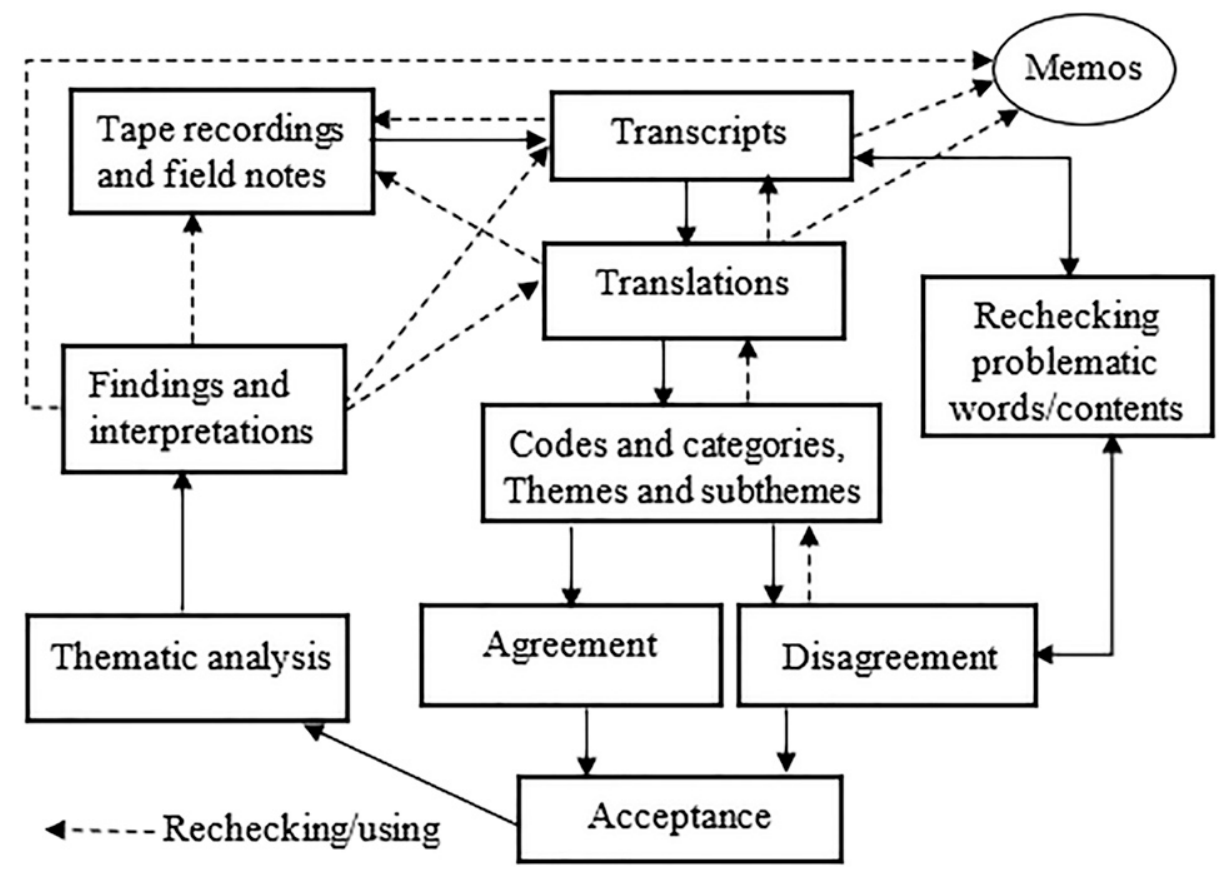

FIGURE 1. Iterative steps of data transcription, translation, analysis, and interpretation of findings on the barriers to TB case detection in East Gojjam zone of Ethiopia, 2018.

7. Interpret the findings by rechecking the audio records, transcripts, translations, and memos.

The transcribed data were analyzed thematically as per the Braun and Clark ${ }^{20}$ approach using QS NVivo 12 software program. The investigators read and reread the transcripts of IDIs and FGDs to understand the data and generate initial codes to the texts. Through a series of discussions and readings, those initial codes were refined and grouped through axial coding into categories. Then, the categories were organized into main and subthemes through several iterations based on concept similarities. The analysis was concluded after a thorough discussion among the investigators. The findings are presented in narration with introductory descriptions followed by selected respondent quotes using their own words to give more insights into the barriers to TCD. The quotations are presented in italics with certain indents and a source for each quotation.

Ethical considerations. The ethical review committee of the College of Medicine and Health Sciences, Bahir Dar University, reviewed the research proposal and gave an ethical clearance (Protocol No: 091/18-04). We took supportive letters from the Amhara Regional State Health Bureau and East Gojjam Zone Health Department. Data were collected after getting written informed consent including permission to use an audio recorder from each study participant. Participation was voluntary based, and maximum efforts were made to give an equal chance to all participants to participate and respect others' ideas during the FGDs. Interviews were conducted in quiet private offices arranged for the interview to avoid any disturbance and keep participants' privacy. Data privacy and confidentiality were maintained through anonymity and restricting access to data.

\section{RESULTS}

Characteristics of study participants. Of the 67 study participants, 39 (58.2\%) were males, 33 (49.3\%) had first- degree education, and 44 (65.7\%) were urban residents. The mean age of the respondents was $36.46(\mathrm{SD} \pm 7.4)$ years and $83.6 \%$ of them were within the age-group of 25-44 years. Forty-eight $(71.6 \%)$ participants were government employees, and $40(60 \%)$ were HWs working in health facilities (Table 1).

Barriers to TB case detection. All the study participants agreed that the overall TCD was low in East Gojjam zone.

TABLE 1

Descriptions of study participants in East Gojjam zone of Ethiopia, 2018

\begin{tabular}{lcc}
\hline \multicolumn{1}{c}{ Variable } & TB patients, $n$ (\%) & TB officer/HW, $n$ (\%) \\
\hline Age (years) & & \\
$25-34$ & $14(21.0)$ & $14(21.0)$ \\
$35-44$ & $5(7.4)$ & $23(34.3)$ \\
$45-54$ & $1(1.5)$ & $9(13.3)$ \\
$\geq 55$ & $1(1.5)$ & $0(0)$ \\
Gender & & \\
Male & $11(16.4)$ & $28(41.8)$ \\
Female & $10(15.0)$ & $18(26.8)$ \\
Education level & & \\
No formal education & $8(11.9)$ & $\mathrm{NA}$ \\
Primary school & $6(9.0)$ & $\mathrm{NA}$ \\
High school & $4(6.0)$ & $\mathrm{NA}$ \\
College (diploma) & $0(0)$ & $12(18.0)$ \\
University (Bachelor's degree) & $3(4.4)$ & $30(44.7)$ \\
University (Master's degree) & $0(0)$ & $4(6.0)$ \\
Residence & & \\
Rural & $7(10.4)$ & $16(23.9)$ \\
Urban & $14(21.0)$ & $30(44.7)$ \\
Occupation & & \\
Farmer & $5(7.4)$ & $\mathrm{NA}$ \\
Government employed & $2(3.0)$ & $46(68.6)$ \\
Driver & $4(6.0)$ & $\mathrm{NA}$ \\
Merchant & $4(6.0)$ & $\mathrm{NA}$ \\
Student & $2(3.0)$ & $\mathrm{NA}$ \\
Unemployed & $4(6.0)$ & $\mathrm{NA}$ \\
\hline NA = not applicable. & &
\end{tabular}


Tuberculosis patients and HWs identified several barriers affecting the performance of TCD that are grouped into three main themes and 14 subthemes. The main themes were 1) patient-related barriers, 2) health system-related barriers, and 3) sociocultural and environmental barriers (Figure 2).

Theme 1: Patient-related barriers. This theme contains barriers that have challenged individual TB patients from timely health seeking for TB. The theme is further categorized into three subthemes: rural residence and visit to nonformal health providers, poverty, and low TB knowledge and practice (Figure 2).

Rural residence and visit to nonformal health providers. Most of the study participants explained that poor access to TB diagnostic services in rural areas influenced TCD performance.

"People from rural areas do not get timely TB services due to the inaccessibility of TB diagnostic facilities. Their place of residence also affects their awareness and healthseeking behavior, and treatment adherence. In addition, patients' experience of delays in getting proper services at their pre-scheduled appointment time at health facilities leads them to stay at home or visit traditional healers or drug stores available in their vicinity. This results in long patient delay which ultimately leads to low TB case detection." (A 42-year-old priest TB patient)

Similarly, TB control program officers also described the negative impact of rural residence on TCD.

"People from rural settings are not getting adequate healthcare services for TB. The availability of TB diagnostic services, health literacy of the population and geographic location of health facilities affect TB case detection performance. People residing in rural areas lack appropriate information sources about TB, means of transportation, and communication options. All these conditions may contribute to an increased number of undetected infectious TB cases in the community. Place of residence plays an important role in TB case detection activities since about $85 \%$ of the population is living in rural areas." (38-year-old and 42-year-old male TB officers)

Furthermore, all the study participants noted that long distance from the health facilities and patient delays to visit health facilities were major barriers that caused low TCD performance in the study area.

"Many TB patients visited formal health facilities after using all the possibilities at their disposal [taking homemade remedies, visiting traditional healers, spiritual services, and drug stores]. They even visited these places after a long delay. An increased number of TB cases are detected in our facilities during none harvesting times. Distance from the health facilities, low awareness of TB, poor income, absence of transportation access, and visiting informal health services providers are factors for patient delays." (A 42-year-old female HW: FGD6)

Poverty. Almost all respondents noted that poor income impeded people from early healthcare-seeking practice. They mentioned that poor income is the root cause of long patient delay.

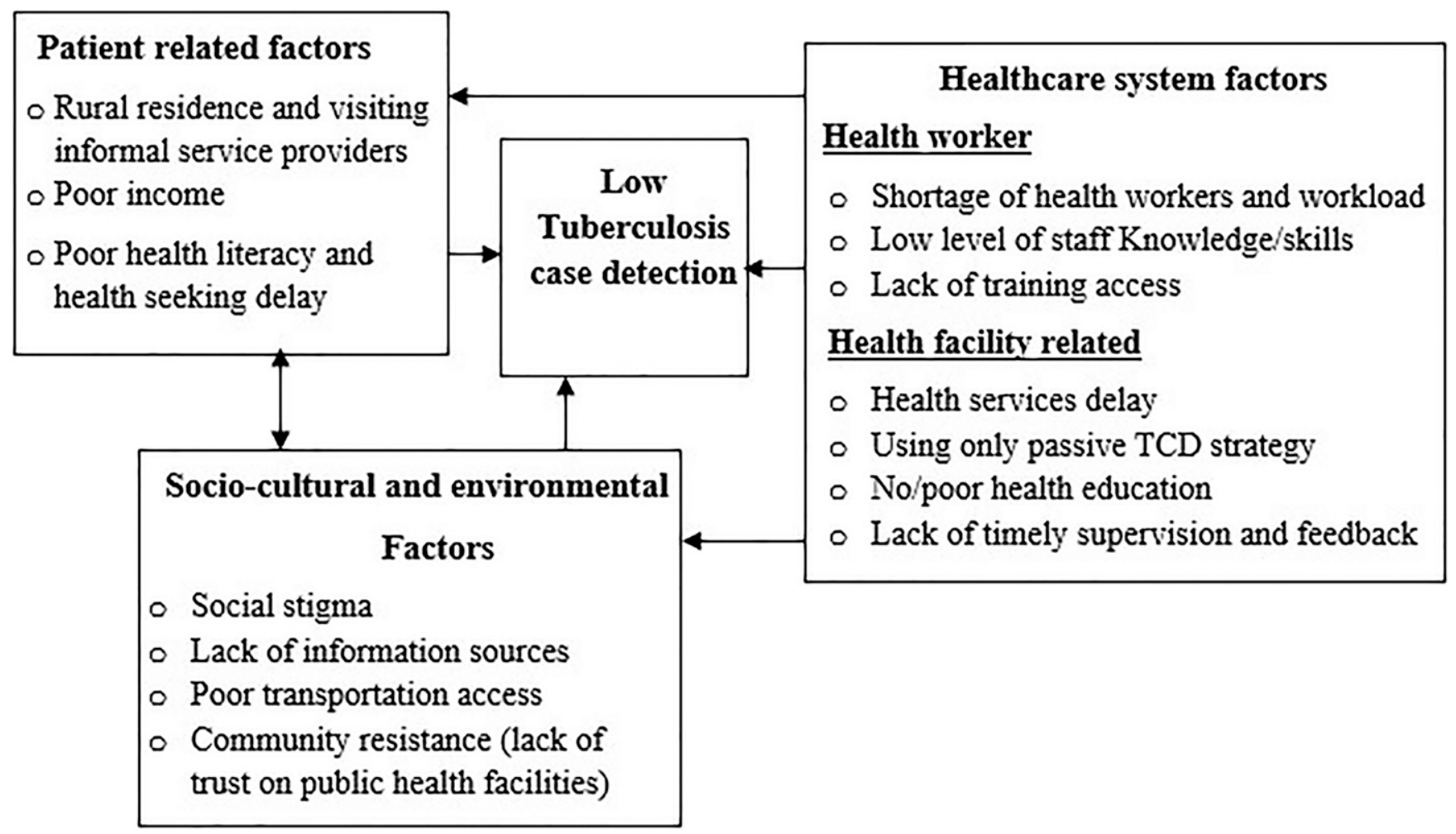

FIGURE 2. Main and subthemes about the barriers to TB case detection (TCD) in East Gojjam zone of Ethiopia, 2018. 
"Poor income is a major barrier for timely health-seeking and TB diagnosis. Patients with poor income may not get money for transportation, and to cover other costs related to their illness. Due to this, most infectious TB cases stay at home until getting money and social supports. This will increase disease complication, and transmission to others." (A 37-year-old female TB patient)

Health workers also explained the influences of poor income on TCD as follows.

"Poor income is the main barrier of lowering TB case detection by preventing people from early health seeking for TB diagnosis. This happens due to high cost for transportation, various medical expenses, and accommodation costs during diagnosis, check-ups and collecting anti-TB drugs. Also, poor income is a key factorfor undernutrition that facilitates the risk of acquiring TB disease and complications of illnesses. All these contribute to low TB case detection, long infectious period, disease complication, absence from work, and death." $(A$ 56-year-old male HW: FGD3)

Poor TB knowledge and health-seeking delay. All TB patients and most of the HWs mentioned that low community awareness about TB and giving priority to their day-to-day routine tasks among patients were barriers to enhanced TCD. They noted that the absence of adequate information sources for TB is a key factor for poor awareness. Over half, 12 (57\%), of the interviewed TB patients had the wrong perceptions about the cause of TB.

"TB is a disease caused by cold air, high workload, hunger/poverty/, and overthinking. I got TB since I was so busy with my routine activities [harvesting time and have no supporters]. I was exposed to cold air while I was performing my routine tasks since early in the morning. I even did not take proper food. I went to a health center after two months of the onset of my illness (cough) following the advice I got from a TB patient who came to visit me at my home." (A 38-year-old male farmer TB patient)

Health workers also described contributing factors to poor awareness and its effects on TCD.

"People's preferences not to open windows when traveling by taxi or on public transport, and closing of windows at home and workplaces, long patient delays, first visit to traditional healers, and relating the cause of their cough to a common cold, pneumonia, workload, and cold air are indicators of low community awareness about TB. It is because of lack of education, limited information sources, and providing less attention to health literacy that TB patients have poor knowledge about TB which leads to low TB case detection." (A 38-year-old male HW: FGD5)

Theme 2: Sociocultural and environmental barriers. This theme contains barriers to TCD that originated from the sociocultural facets of the environment where the patients are residing. The theme contains two subthemes (Figure 2).
Stigmatization. During the interviews and discussions, most of the participants considered social stigma as an important barrier to TCD.

"People are not interested to live and take part in any social activities with TB patients. People including intimate friends and neighbors show unusual reactions when meeting TB patients. They cover their mouths with clothes, talk to each other about us, and limit their contact with us after knowing our TB status. Most TB patients have decided not to be part of any social event, and have limited their contact with their friends. I have experienced this problem and decided to limit myself from interacting with my friends and attending social events." (A 37-year-old female TB patient)

Most of the HWs also described the influence of stigma on TCD.

"Several TB cases have visited traditional healers, and spiritual services to get relief from their illness (cough) before visiting health facilities. Cultural beliefs and practices in the community push patients to visit non-formal health providers. As a result, many patients with cough are not visiting health facilities. In addition, confirmed TB cases do not want to disclose their status because of fear of stigma. This leads to patient delay and low TB case detection." (A 52-year-old male HW: FGD3)

Similarly, HEWs stated the challenges of stigmatization on TCD as follows:

"Many people in the rural community are unwilling to reveal the presence of any person with cough in their family (households). If a TB suspect is identified in the household, they do not accept any referral for TB diagnostic services. This is related to various factors including fear of stigma and social discriminations and perceived fear being diagnosed for HIVIAIDS/TB coinfections." (A 36-year-old HEW: FGD1)

Low community trust in the available health services. Most of the study participants reported that low community trust in the health service provided by HEWs is an important barrier to improved TCD performance. In particular, "educated" people in the community tend to not listen to HEWs' advice assuming that they have better knowledge than the HEWs.

"To date, HEWs are not providing healthcare services as before. Mostly, they are absent from their office and even their presence at office is just for reporting. Moreover, due to inadequate TB control services, there is no regular provision of health education about TB. There is unexpected delay of TB diagnosis results for samples sent to referral sites. TB suspect samples are usually lost in referral sites and resampling request is common. HEW services do not cover all localities. At present, many people have no confidence and trust on HEWs professional practices." (A 30-year-old BSc-completed female TB patient)

The HWs and TB control officers also stated the status of community acceptance to HEWs and its impact on the TB control program performance. 
"Inadequate performance in TB suspect identification and referral has affected our TB case detection achievement in our catchment area. Also, community residents who have better education do not listen to our advice assuming that they are better than us. Feelings of demotivation among HEWs and the absence of household members at the time of HEW visit negatively affect our effort to improve TB case detection." (32-year-old HEW and 48-year-old female TB officer)

Absence or poor means of transportation access. Most of the study participants indicated that shortage of means of transportation is a major barrier to access TB diagnosis and treatment services in East Gojjam zone, mainly in rural and remote areas where road infrastructures are very limited.

"There is no transportation access for most of the community members in the rural and remote areas due to poor road infrastructures. Many people have used stretchers made from local materials to transport patients to healthcare services. This wastes patients' and caregivers' working times as they have to use days to weeks for travel and getting healthcare services. Alternatively, many patients are staying at home being ill with possibilities of progressing to severe illness and becoming highly infectious to household members and others who might be in close contact with them. Patients staying at home use homemade remedies since they cannot access means of transportation and the necessary supports." (A 38-yearold male TB patient)

Health workers also stated the impact of poor means of transportation on TB control activities from the patient and health service perspectives.

"Poor transportation access has affected both TB patients and the activities of health service providers. Besides the shortage, the high transportation cost has limited the health-seeking behaviors of rural communities. Most patients have missed their appointments for TB services due to lack of money to cover transportation fees. Also, TB control officers and HWs are not offering outreach TB services, timely supportive supervision, and feedback due to absence or limited transportation access." (34-year-old and 35-year-old female HWs: FGD1 and 6)

Theme 3: Healthcare system-related barriers. This theme includes barriers to enhanced TCD related to the health system. The theme is further grouped into two subthemes: HWand health facility-related barriers (Figure 2).

Health worker-related barriers.

Shortage of HWs and workload. Majority of the study participants noted that workload and manpower shortage, particularly laboratory staff and senior physician shortages as main barriers to improved TCD performance.

"If you visit health facilities in most rural areas, majority of them do not offer TB diagnostic services due to lack of laboratory technicians and skilled health workers. Usually, patients who come with cough are examined and managed by less skilled health workers. These health workers are very few in number and use patients' clinical symptoms to reach at TB diagnosis. This creates workload among staff and results in poor service quality, long health service delay, low patient satisfaction and encourages patients to visit other health facilities that have TB diagnostic service." (A 30-year-old male TB patient)

"Health workers in most health facilities have high workload due to high patient load, lack of adequate number of staff such as laboratory technicians, senior physicians and HEWs. This happened due to the absence of such HWs in the job market as a result of closure of some mid-level training programs (laboratory technicians training), and high staff turnover due to poor management practices and absence of staff motivation strategies." (A 46-year-old male HW: FGD4)

Moreover, respondents noted the presence of high workload among HEWs due to staff shortage. They said that rendering 16 health service packages at a household level with a limited number of HEWs made them busy and less effective in achieving their plans in TCD.

"High workload has impeded community TB case detection since we also have a number of other health service activities in the community. The nature of our work is unique. We regularly do house to house visits, which is very tiresome. The population size is rapidly increasing but the number of HEWs assigned ranges from two to four per health post. Although the standard says one HEW for 5,000 people, one HEW is currently serving for over 10,000 population. We have tried our best, but no progress in TB case detection performance." (34-year-old and 36-year-old HEWs: FGD1 and 2)

Study participants also stated the presence of high workload among HWs working at health institutions that have TB diagnostic services. This is because of staff shortage because they are serving as TB confirmatory testing sites for TB suspect samples referred from the nearby health facilities. This can affect service quality, patient waiting time, and staffpatient interaction.

"Health workers from health facilities that have TB diagnostic services (public and private) have relatively high workload since they are small in number and have to manage high patient and patient sample loads. They provide TB diagnostic services for referred sputum samples and self-referral patients including outside of their catchment areas. These contribute to service delay, provision of poor quality services which ultimately affect the TB case detection performance." (A 40-year-old male $H W: F G D 3)$

Knowledge and skills gaps in HWs. Some of the respondents stated the presence of skill and knowledge gaps among the HWs that lead to poor TB suspect screening and frequently managing patients with broad-spectrum antibiotics. 
They said that these were the main causes of poor patient satisfaction and trust in public health facilities. The following quotes from participants clearly illustrate these problems:

"Patients are repeatedly treated with antibiotics which usually leads them to complain because of exacerbation of their symptoms. This will encourage patients to make self-referral decisions to visit other healthcare facilities which are costly. A few days after visiting those health facilities, they come back with a positive TB results. This implies that we are missing infectious TB cases due to knowledge/skill gaps. This usually happens among HWs who have graduated from private colleges. Many people say that HW who graduated from private colleges did not get adequate supportive supervision and had fewer clinical practice hours before graduation." (A 38-year-old male HW: FGD5)

Interviewed TB patients also stated their perceptions about the knowledge, skills, and supportiveness of HWs as follows:

"There was better health service delivery in previous times. HWs were so kind, knowledgeable, accountable, and provided better diagnostic and treatment services. But now, it is the opposite. They do not properly assess and manage patients and do not provide adequate time to exhaustively listen to patients' complaints. They simply order antibiotics without properly diagnosing the disease. That is why we are repeatedly visiting several health facilities without getting improvements in our symptoms. As a result, some patients may go to traditional healers, and others may stay at home." (A 40-yearold male TB patient)

Lack of staff training. Study participants also noted that the limited training access in each health facility is a contributing factor to poor quality of TB care and case detection.

"In-service training plays a key role to update the knowledge and skills of HWs and improve service quality. However, only few health facilities have trained staff on TB. If more staff were trained, there would not be gaps when there is staff turnover. Poor attention from regional, zonal and woreda health managers, scarce and unfair distribution of training opportunities to HWs, and absence of NGOs working on TB are factors to poor training access." (A 46-year-old male health worker: FGD4)

Health facility-related barriers.

Health service delay. The study participants reported that long health service delay is a big challenge contributing to low TCD. Most TB patients knew their status after repeatedly taking antibiotics and visiting several other health facilities.

"Health facilities are not giving timely TB services. Visiting several health facilities, long waiting times, and taking repeated antibiotics prescriptions are common problems. For example, I knew my TB status after visiting three health facilities in five months time period since my first facility visit." (A 25-year-old female farmer TB patient)
"Health service delay is a big challenge in majority of the rural health centers. The trend of repeated use of antibiotics, low level of knowledge/skill among HWs, lack of TB diagnostic facilities, poor staff commitments, and lack of timely feedback from sample referral sites are contributing to health service delay. All these result in poor patient satisfaction and lead patient to visit informal health service providers." (A 36-year-old male HW: FGD3)

Health extension workers also described the long service delay and its impact on their day-to-day activities as follows:

"The main cause of TB service delay is the delayed feedback from the referral testing sites (health facilities that have TB diagnostic services) mainly due to high workload, loss of referred samples at the testing centers, and sample rejection for poor sample preparation and transportation procedures. As a result, patients have started practicing visiting non-formal service providers i.e. self-referral to other health facilities by passing HEWs. This has an impact on our routine healthcare activities." (A 33-year-old HEW- FGD1)

Using only passive case detection strategy. Relying only on passive TCD was reported as a potential barrier to low TCD performance. The study participants agreed on the importance of considering an active TCD approach because of the lack of TB diagnostic services in rural health facilities. They described the importance of active TCD as follows:

"Our TB case detection performance will not be improved unless we supplement the passive case detection strategy with active case detection approach in congregated places and population groups [prisons, schools, among homeless people, close contacts and remote woredas]. We practiced this when I was working in a rural health center and got an increased number of TB positive cases. The neighboring woreda manager also took that as a lesson and practiced it in his woredas and got better feat in TB case detection." (A 36-year-old male HW: FGD5)

Absence or poor health education performance. Study participants stated that most of the health facilities were not providing regular health education to the patients and caregivers. Some health facilities have no health education program. The following quotes explain the status of health education in health facilities.

"People from rural areas, do not have awareness about TB. Most people are illiterate and have no access to TVI radio. HEWs and HWs are not regularly giving health education. People get information about TB when they develop TB and have contact with TB patients who are on anti-TB treatment." (A 40-year-old male merchant TB patient)

"We are not regularly offering health education. Even, the way we deliver health education is not properly framed in a way that is suitable for the needs of illiterate people. The 
equality of health education given is not monitored by the concerned health officials. There is lack of appropriate teaching aid. There is also resistance to health education from patients and caregivers since they give more attention to treatment rather than on prevention of TB." (38year-old female HW: FGD6)

Lack of supervision and feedback. Majority of the HWs mentioned the absence of supportive supervision and timely feedback as a potential barrier hindering the improvement of TCD (Figure 2) performance. They reported that the way supportive supervision activity conducted in health posts is weak, irregular, and timely feedback is not provided.

"Conducting supervision and providing timely feedback to HEWs is the duty of health center staff. But their supervision is not satisfactory and is more of criticism with no feedback. Due to the delayed feedback from health centers about the status of the TB suspects referred for TB diagnosis, the community has developed mistrust towards health centers and has become resistant towards HEWs activities in the community, particularly on TB suspect identification and sputum sample referral. It has become a serious issue for our TB case detection performance and other activities." (A 36-year-old HEW: FGD1)

"We cannot say there are regular supportive supervision activities and provision of timely feedback these days. The supervision conducted is irregular, traditional, and is done for the sake of reporting purposes. This might be related to lack of paying, poor commitment of the concerned staff, lack of budget, poor program integration, and lack of means of transportation." (A 36-year-old male HW: FGD5)

Contrary to the aforementioned themes, one regional and one zonal level TB control program officers stated that the primary reason for low TCD performance is the decline of TB incidence in the community which is related to better access to TB services, laboratory reagents, and supplies; increased community awareness; and improved health-seeking behavior of patients.

"TB case detection is lower in rural than in urban areas. From my work experience in rural health facilities, the real cause for the current low TB case detection performance is the decline of TB incidence in the community due to the high efforts of HEWs, HWs in the health facilities, NGOs and TB officers. The community awareness about TB transmission and prevention has also improved. However, there are more undetected TB cases in urban areas due to high population crowding and mobility [drivers, daily laborers, and merchants], homeless people and beggars], poor living conditions, poor income, the existence of comorbidities associated with TB [HIV and DM], and miss trust of HEWs." (37-year-old and 42-year-old female TB officers)

\section{DISCUSSION}

This is the first study that combines the perspectives of patients, HWs, and TB control program officers in understanding the barriers to TCD in East Gojjam zone, Ethiopia. All the study participants noted that early case detection is crucial to expedient treatment and reduce TB transmission. Study participants also discussed on the presence of varied TCD achievements across health facility types and locations. Although some study participants stated the presence of relatively better TCD in a few urban health facilities, most of them agreed that many health facilities, mainly from rural areas, had poor TCD performance, and even some had no TB diagnostic services. The study participants discussed potential barriers that need to be given due attention to improve the current low TCD in the study area. This discussion follows the list and order of themes and subthemes depicted in Figure 2 including TB patient-related barriers, sociocultural and environmental barriers and healthcare facility-related barriers.

Patient-related barriers. In this study, rural residence was a key barrier to enhanced TCD. Majority of the study participants noted that many health facilities in the rural and remote areas do not have AFB smear microscopy testing mainly because of the lack of laboratory staff and the necessary laboratory reagents and supplies. This result agrees with findings from previous studies ${ }^{4,12,18}$ and implies inadequate access to TB diagnostic services in rural areas where $85 \%$ of the study population is residing. ${ }^{9,23}$

Poor income of TB patients was also identified as a barrier to TCD. During the discussions, most participants raised the presence of undetected TB cases in remote areas due to the inability of patients to cover costs for transportation, accommodation fees, and other related costs incurred while seeking care at the $\mathrm{HC}$ or hospital. Similar findings were reported from former studies where financial burdens were barriers to access TB services. . $^{3,24-26}$

In addition, community awareness about TB was mentioned as a potential barrier that impedes TCD practices. During the interview, over half $(57 \%)$ of the TB patients provided the wrong answer for the cause of TB. This was supported by an earlier study finding from Ethiopia ${ }^{27}$ where $74.2 \%$ of the study population did not know the cause of TB infection. Low TB awareness usually makes the community link their cough with a common cold, pneumonia, asthma, and high workload and lead them to decide either to stay at home taking home-based remedies or visit traditional healers, spiritual places, and local drug stores to treat their symptoms. ${ }^{7,25,27}$ These result in long patient delays to TB diagnosis. Several studies conducted on healthcare system's delays reported that poor community awareness was a key barrier to diagnostic delays. , $^{3,26-29}$ This was related to various contributing factors including low performance of HEWs in providing health education, poor health education programs at health facilities, community resistance to health education, lack of paying attention to health education by TB control staff, and limited access to health information sources. ${ }^{26,30}$ The absence of teaching aids, not considering health education as part of the routine health service activities, and workload among HWs might also contribute to absence or poor health education practice at healthcare facilities. ${ }^{18,23,30}$

Health service-seeking delays and giving less priority to their illness by patients were identified as barriers to TCD. This finding is similar to other studies that indicated the level of sickness as a determinant factor for long delays among remote communities with poor health literacy. ${ }^{4,26,31}$ Inaccessibility of TB diagnostic services, ${ }^{23,24,32,33}$ severity of illness, 
fear of stigmatization, ${ }^{3,28,33}$ and visiting traditional healers ${ }^{10,23}$ might have a role in the long health service-seeking delay.

Sociocultural and environmental barriers. Most of the study participants also described cultural influences (social stigma) affecting TCD activities. Similar studies from different areas reported the impact of stigmatization on TB control activities. ${ }^{9,26,28}$ This indicates that there could be undetected TB cases in the community because of fear of stigma and discrimination from close friends and community members. The effect of stigma is observed both before and after diagnosis of TB. A study showed that several confirmed TB cases did not disclose their TB status and tended to misinform the type of drug they were taking as antimalarial or pneumonia drug. ${ }^{34}$ Also, patients sometimes get difficulties to accept their status and decide either to take spiritual services or to visit health facilities far from their residential area and get anti-TB treatment. This is intended to minimize disclosure of their TB status to their friends and other community members who know them well. ${ }^{30}$ All these situations create additional psychological stress, poor treatment adherence, and loss of family or social supports. Many people in the community also think that TB and HIV/AIDS are inseparable. For these reasons, most patients prefer visiting informal service providers compared with the formal ones. ${ }^{4,10}$ This results in a long infectious period and leads to low performance of TCD.

Transportation shortage was identified as a barrier contributing to low TCD performance in the study area. Poor transportation facilities can affect the activities of both the community and the TB control staff. Because of the poor transportation infrastructure and high cost for transport, many TB patients may either delay in seeking care or decide to not visit formal health facilities for diagnostic services. Equally, it can hinder timely delivery of the necessary laboratory reagents and supplies to health facilities. Poor transportation infrastructure can also prevent TB control program staff from providing training and regular supportive supervision at remote health facilities. Earlier studies showed that poor transportation infrastructure resulted in high patient delays, poor facility readiness, high staff turnover, and poor service quality. $3,4,16,25$

Low community trust in health services including resistance to health education and referral services for TB mainly from the "educated" and government employees has challenged HEWs and HC staff activities. The reasons for this might be linked to poor awareness of the community about TB, being busy with routine tasks, or less confidence in the knowledge and skills of HEWs and $\mathrm{HC}$ staff. ${ }^{27,30}$ This implies that there is a need for intervention to solve such disagreements by designing the appropriate health education and behavioral change communication strategies for TB.

Healthcare system barriers. The study participants highlighted the shortage of laboratory technicians, senior physicians, and HEWs, particularly in rural health facilities. Findings from previous studies support this result. ${ }^{3,12,33-35}$ This indicates that many health facilities in rural areas are not offering TB diagnostic services because of shortage of staff. The possible reasons for this gap are the absence of new graduates in the job market and high staff turnover due to poor management and absence of staff motivation strategies. ${ }^{18,19,36}$ Tuberculosis control without effective trained staff and TB diagnostic facility is impossible. Therefore, it is recommended that the local health officials understand the challenge and devise appropriate strategies to solve the problem and enhance the TCD performance.

As a result of staff shortage and poor readiness of health facilities, high workload was mentioned among HWs as a barrier that contributed to low TCD. This happened because of the inadequate number of health staff available in the health facilities as compared with the national standard that defines the acceptable number of healthcare professionals per health facility. ${ }^{23}$ In particular, those healthcare centers that serve as TB suspect identification and patient referral facilities were the most affected. This finding was supported by various study results from different places. ${ }^{12,17,23,35}$

Poor knowledge and skill gaps among HWs were identified as barriers affecting TCD. Knowledge and skill gaps among HWs contribute to low index of suspicion, which results in missing potential TB cases at health facilities and community levels. This finding is in line with former studies that reported staff skill and knowledge gaps as barriers to improved quality TB care. ${ }^{12,19,30,33}$ The absence of in-service training to HWs and poor staff recruiting process might contribute knowledge and skill gaps.

In-service training is crucial to update knowledge and skills of HWs. ${ }^{37}$ However, large numbers of study participants reported the presence of poor training access in East Gojjam zone. This indicates that HWs are working only based on the knowledge and skills they have got from their undergraduate studies at colleges or universities. This will have a direct impact on the quality of TB diagnosis and treatment services. Previous studies indicated the absence of in-service training to HWs. ${ }^{18,19,23,26,37}$ The causes for the lack of or poor inservice training might be related to the absence of NGOs, inadequate budget allocation, less attention paid by concerned health officials, and unfair distribution of training. ${ }^{37}$

Although most of the health facilities used a sample referral system as an alternative for TB diagnostic services, they were unable to satisfy their patients. This is because of a long health service delay, loss of sample, and sample rejection at the testing sites due to poor sample preparation and sample transportation qualities. Sample loss will lead to the recollection of samples which causes mistrust among communities toward public health facilities. This will encourage patients to opt for self-treatment, visit traditional healers, or self-referral to health facilities from other places. All these contribute to long TB diagnostic delays and poor TCD performance. 9,24,26,28,33

Study participants also believed using only a passive TCD strategy had played a role in lowering TCD performance. Because of poor transportation access, low income, poor TB awareness, less accessible geographic locations, low HEWs performance, and cultural influences, most of the rural residents have poor healthcare service-seeking behaviors. The local TB control program and health facility staff need to consider an alternative TCD strategy, an active TCD approach, particularly for those people and elders who do not have supporters. ${ }^{16,31,38,39}$

Regular supportive supervision and provision of feedback is the most effective TB control program monitoring strategy. Timely feedback in the form of reports, technical supports, and provision of training after supervision are important for HWs to take corrective actions and improve TCD. Conversely, all HWs stated that supportive supervision is rarely practiced in the study area and no timely feedback is provided. A former study supports this result where timely supervision and feedback were barriers to facility readiness. ${ }^{23}$ Most HEWs mentioned 
that they encountered resistance while offering referral paper to patients who had TB signs and symptoms. There is a long TB diagnosis delay including sample loss. This clearly indicates the need for regular supportive supervision and feedback for taking timely corrective actions. The absence of regular supervision and feedbacks along with community resistance and poor staff commitment results in low performance of community TCD. This finding agrees with results of former Ethiopian study where HEWs contributed less in awareness creation and referral of TB suspects. ${ }^{29}$

On contrary, two TB control program officers differently stated their views on the status of TCD and potential barriers. They stated that TCD is lower in rural than that in urban areas. The reason they mentioned was the decline of new TB cases/ incidence in rural areas as a result of improved access to health services and effective TB control activities at the community levels, but they accepted the presence of low TCD in urban areas for various reasons. From this, we can understand the presence of discrepancies between the views of TB control program officers and HWs on TCD which is unexpected. Also, the $\mathrm{WHO},{ }^{1}$ national and regional TB reports, ${ }^{40,41}$ and various studies $^{8,10,19,42}$ revealed the presence of low TCD and more undetected infectious TB cases in rural settings.

The strength of this study relies on using triangulation of data sources and data collection methods to address the objectives of the study. In addition, the study has identified several barriers affecting enhanced performance of TCD by involving potential stakeholders. The study could have come up with more information if it had included selected community members, traditional healers, and other nonformal health providers.

\section{CONCLUSION}

This study revealed that the TCD activity which is one of the pillars of the TB control program has been confronted with several patient-related, environmental, and healthcare system-related barriers. Among others, rural residence, poverty, low community awareness, social stigma, shortage of health information sources, community resistance, poor transportation infrastructure and access, shortage of HWs, low level of staff knowledge and skills, lack of training access, patient and health service delay, lack of supportive supervision and timely feedback, and poor health education practice in healthcare facilities were barriers affecting enhanced TCD performance in the study area. Increasing health literacy of the community through well-crafted health education and behavioral change communication strategies, improving access and quality of TB diagnostic services, conducting regular supportive supervision and provision of timely feedback, arranging regular refresher training and devising attractive staff motivation and recruitment schemes, engaging local health officials and political leaders to address budgetary problems and transportation infrastructure challenges are imperative interventions to enhance the TCD efforts in the study area.

Received January 19, 2020. Accepted for publication June 14, 2020. Published online August 3, 2020.

Acknowledgments: We would like to express our deepest gratitude to Bahir Dar University for financing this study. We are also grateful to the staff of the Amhara Regional State Health Bureau and East Gojjam Zone Health Department, study participants, data collectors, and supervisors for their candid support during data collection. The
American Society of Tropical Medicine and Hygiene (ASTMH) assisted with publication expenses.

Financial support: Bahir Dar University covered the budget for data collection (Grant No: RCS/254/09), but it has no technical role in all the research processes.

Authors' addresses: Mulusew Andualem Asemahagn, School of Public Health, College of Medicine and Health Sciences, Bahir Dar University, Bahir Dar, Ethiopia, E-mails: muler.hi@gmail.com ormulusew. andualem@bdu.edu.et. Getu Degu Alene, Department of Epidemiology and Biostatistics, School of Public Health, School of Public Health, College of Medicine and Health Sciences, Bahir Dar University, Bahir Dar, Ethiopia, E-mail: adgetu123@yahoo.com. Solomon Abebe Yimer, Department of Microbiology, Unit for Genome Dynamics, Faculty of Medicine, University of Oslo and Coalition for Epidemic Preparedness Innovations (CEPI), Oslo, Norway, E-mail: adgetu123@yahoo.com.

\section{REFERENCES}

1. World Health Organization, 2019. WHO Global Tuberculosis Report 2019. Geneva, Switzerland: World Health Organization. Licence: CC BY-NC-SA 3.0 IGO.

2. Duko B, Bedaso A, Ayano G, Yohannis Z, 2019. Perceived stigma and associated factors among patient with tuberculosis, Wolaita Sodo, Ethiopia: cross-sectional study. Tuberc Res Treat 2019: 5917537.

3. Soomro MH, Qadeer E, Mørkve O, 2013. Barriers in the management of tuberculosis in Rawalpindi, Pakistan: a qualitative study. Tanaffos 12: 28-34.

4. Tadesse T, Demissie M, Berhane Y, Kebede Y, Abebe M, 2013. Long-distance traveling and financial burdens discourage tuberculosis DOTs treatment initiation and compliance in Ethiopia: a qualitative study. BMC Public Health 13: 424.

5. Dheda $\mathrm{K}$ et al., 2017. The epidemiology, pathogenesis, transmission, diagnosis, and management of multidrug-resistant, extensively drug-resistant, and incurable tuberculosis. Lancet Respir Med 5: 291-360.

6. McLaren ZM, Sharp AR, Zhou J, Wasserman S, Nanoo A, 2017. Assessing healthcare quality using routine data: evaluating the performance of the national tuberculosis program in South Africa. Trop Med Int Health 22: 171-179.

7. Abebe $\mathrm{G}$ et al., 2012. Tuberculosis lymphadenitis in Southwest Ethiopia: a community based cross-sectional study. BMC Public Health 12: 504.

8. Asemahagn MA, 2017. Are shopkeepers suffering from TB infection in Bahir Dar city, Northwest Ethiopia. Tuberc Res Treat 2017: 2569598

9. Asemahagn MA, Alene GD, Yimer SA, 2019. Tuberculosis infectious pool and associated factors in East Gojjam Zone, Northwest Ethiopia. BMC Pulm Med 19: 229.

10. Asres M, Gedefaw M, Kahsay A, Weldu Y, 2017. Patients' delay in seeking healthcare for tuberculosis diagnosis in East Gojjam Zone, Northwest Ethiopia. Am J Trop Med Hyg 96: 1071-1075.

11. EFMoH, 2015. Health Sector Transformation Plan (HSTP): 2015/ 16-2019/20. Addis Ababa, Ethiopia: Ethiopian Federal Ministry of Health.

12. Dabaro D, 2017. Factors affecting tuberculosis case detection in Kersa woredas, South West Ethiopia. J Clin Tubercu Mycobact Dis 9: 1-4.

13. EFMoH, 2018. National Tuberculosis Annual Report and Review Meeting. Addis Ababa, Ethiopia: Ethiopian Federal Ministry of Health.

14. Amhara Regional Health Bureau, 2018. Regional Health Bureau Annual Performance Report. Bahir Dar, Ethiopia: ARHB.

15. East Gojjam Zone Health Office, 2018. The 2018 Annual Performance Report of East Gojjam Zone Health Office. Debre Markos, Ethiopia: East Gojjam Zone Health Office.

16. Cowan J, Greenberg Cowan J, Barnhart S, Demamu S, Fiseha D, Graham W, Melese E, Reason L, Tefera Asfaw F, Feleke G, 2013. A qualitative assessment of challenges to tuberculosis management and prevention in Northern Ethiopia. Int J Tubercu Lung Dis 17: 1071-1075.

17. Workneh MH, Bjune GA, Yimer SA, 2017. Prevalence and associated factors of tuberculosis and diabetes mellitus comorbidity: a systematic review. PLoS One 12: e0175925. 
18. Gebreegziabher SB, Yimer SA, Bjune GA, 2016. Qualitative assessment of challenges in tuberculosis control in West Gojjam Zone, Northwest Ethiopia: health workers' and tuberculosis control program Coordinators' perspectives. Tuberc Res Treat 2016: 2036234.

19. Ereso BM, Yimer SA, Gradmann C, Sagbakken M, 2020. Barriers for tuberculosis case finding in Southwest Ethiopia: a qualitative study. PLoS One 15: e0226307.

20. Braun V, Clarke V, 2006. Using thematic analysis in psychology. Qual Res Psychol 3: 77-101.

21. Creswell JW, 2014. Research Design: Qualitative, Quantitative, and Mixed Methods Approach, 4th edition. Lose Angeles (USA), London (UK), New Delhi (India), Singapore: SAGE Publications Inc.

22. Tong A, Sainsbury $P$, Jonathan C, 2007. Consolidated criteria for reporting qualitative research (COREQ): a 32-item checklist for interviews and focus groups. Int J Qual Health Care 19: 349-357.

23. Asemahagn MA, Alene GD, SA Y, 2020. Geographic accessibility, readiness, and barriers of health facilities to offer tuberculosis services in East Gojjam Zone, Ethiopia: a convergent parallel design. Res Rep Trop Med 11: 3-16.

24. Hailu HA et al., 2020: Patients' satisfaction with clinical laboratory services in public hospitals in Ethiopia. BMC Health Serv Res 20: 13.

25. Awoke N, Dulo B, Wudneh F, 2019. Total delay in treatment of tuberculosis and associated factors among new pulmonary TB patients in selected health facilities of Gedeo Zone, Southern Ethiopia, 2017/18. Interdiscip Perspect Infect Dis 2019: 2154240.

26. Cattamanchi A, Miller CR, Tapley A, Haguma $P$, Ochom E, Ackerman S, Davis JL, Katamba A, Handley MA, 2015. Health worker perspectives on barriers to delivery of routine tuberculosis diagnostic evaluation services in Uganda: a qualitative study to guide clinic-based interventions. BMC Health Serv Res 15: 10.

27. Datiko DG, Habte D, Jerene D, Suarez P, 2019. Knowledge, attitudes, and practices related to TB among the general population of Ethiopia: findings from a national cross-sectional survey. PLoS One 14: e0224196.

28. De Schacht C, Mutaquiha C, Faria F, Castro G, Manaca N, Manhica I, Cowan J, 2019. Barriers to access and adherence to tuberculosis services, as perceived by patients: a qualitative study in Mozambique. PloS One 14: e0219470.

29. Asres A, Jerene D, Deressa W, 2018. Delays to treatment initiation is associated with tuberculosis treatment outcomes among patients on directly observed treatment short course in Southwest Ethiopia: a follow-up study. BMC Pulm Med 18: 64.

30. Getnet F, Hashi A, Mohamud S, Mowlid H, Klinkenberg E, 2017. Low contribution of health extension workers in identification of persons with presumptive pulmonary tuberculosis in Ethiopian Somali region pastoralists. BMC Health Serv Rese 17: 193.

31. Lorent N, Choun K, Malhotra S, Koeut P, Thai S, Khun KE, Colebunders R, Lynen L, 2015. Challenges from tuberculosis diagnosis to care in community-based active case finding among the urban poor in Cambodia: a mixed-methods study. PLoS One 10: e0130179.

32. Tadesse T, Demissie M, Berhane Y, Kebede Y, Abebe M, 2015. The clustering of smear-positive tuberculosis in Dabat, Ethiopia: a population-based cross-sectional study. PLoS One 8: e65022.

33. Amenuvegbe GK, Francis A, Fred B, 2016. Low tuberculosis case detection: a community and health facility-based study of contributory factors in the Nkwanta south woredas of Ghana. BMC Res Notes 9: 330.

34. Tariquzzamana S, McKague K, 2018. Knowledge, attitude and practice, and service barriers in a tuberculosis program in Lakes state, South Sudan: a qualitative study. South Sudan Med J 11: 4-7.

35. EPHI, EFMoH, 2016. WHO: Ethiopian Service Availability and Readiness Assessment Summary Report. Addis Ababa, Ethiopia: Ethiopian Public Health Institute (EPHI). Available at: https:// www.ephi.gov.et/images/pictures/download2009/v6\%20Final\% 20SARA\%20Report\%20Jan\%202017.pdf. Accessed October 21, 2018.

36. Deriba BK, Sinke SO, Ereso BM, Badacho AS, 2017. Health professionals' job satisfaction and associated factors at public health centers in West Ethiopia. Hum Resour Health 15: 36.

37. Kuupie D, Adu KM, Apiribu F, Bawontuo V, Adogboba DA, Ali KT, Mashamba-Thompson TP, 2019. Geographic accessibility to public health facilities providing tuberculosis testing services at point-of-care in the upper east region, Ghana. BMC Public Health 19: 718.

38. Yassin MA et al., 2013. Innovative community-based approaches doubled tuberculosis case notification and improve treatment outcome in Southern Ethiopia. PLoS One 8: e63174.

39. Datiko DG, Guracha EA, Michael E, Asnake G, Demisse M, Theobald S, Tulloch O, Yassin MA, Cuevas L, 2019. Subnational prevalence survey of tuberculosis in rural communities of Ethiopia. BMC Public Health 19: 295.

40. Fuady A, Houweling TAJ, Mansyur M, Burhan E, Richardus JH, 2019. Effect of financial support on reducing the incidence of catastrophic costs among tuberculosis-affected households in Indonesia: eight simulated scenarios. Infect Dis Poverty 8: 10.

41. Wynne A, Richter S, Banura L, Kipp W, 2014. Challenges in tuberculosis care in Western Uganda: healthcare worker and patient perspectives. Int J Afr Nurs Sci 1: 6-10.

42. EFMoH and EHNRI, 2011. The First Ethiopian National PopulationBased Tuberculosis Prevention Survey. Addis Ababa, Ethiopia: Ethiopian Federal Ministry of Health. 